\title{
Leveraging Textual Similarity to Predict Beck Depression Inventory Answers
}

\author{
Diego Maupomé, Maxime D. Armstrong, Fanny Rancourt, Marie-Jean Meurs* \\ Université du Québec à Montréal, Montréal, QC, Canada
}

\begin{abstract}
This work proposes an approach to predict potential answers to the Beck Depression Inventory-Second Edition (BDI-II), a 21-item self-report inventory measuring the severity of depression in adolescents and adults. Predictions are based on similarity measures between the textual productions of social media users and completed BDI-IIs. Two methods of establishing similarity are compared. The first one is using unsupervised extraction of topics, and the second one is based on authorship attribution through the use of neural encoders. Both approaches achieve interesting results, indicating that the authorship attribution task can induce a similarity measure useful for depression symptom detection. The issues that arise in predicting several aspects of depression are further discussed.
\end{abstract}

Keywords: Textual similarity - Topic extraction · Authorship attribution - Beck Depression Inventory

\section{Introduction}

Depression is a widespread mental disorder, affecting more than 264 million people of all ages worldwide [1]. The wide spectrum of mental disorders accounts for $13 \%$ of the total global burden of disease, with depression alone representing $4.3 \%$. People with such disorders have disproportionately higher rates of disability and mortality, with up to 40$60 \%$ more chance to die prematurely than the general population [2]. In addition to the threat that mental disorders poses to public health, the economic impact of these is major, resulting in an estimated global economic lost output of US\$16.3 trillion between 2011 and $2030[3]$.

In the United States, around 17.3 million adults had experienced at least one major depressive episode in 2017, representing $7.1 \%$ of all U.S. adult population [4]. In Canada, which is among the few countries where very detailed statistics are available, $20 \%$ of the population will personally experience a mental health issue at any given year. In particular, approximately $8 \%$ of adults will experience major depression at some time in their lives [5]. A recent study by Qin, Wang, and Hsieh [6] shows that the prevalence rate of depression among the adult population in China is estimated as high as $38 \%$.

Despite the proportion of the population affected by mental health issues, and the overall repercussions on society, finding adequate help and resources is burdensome for those who need it, especially for young people. In 2018 , up to $76 \%$ of the care receivers aged 15 to 34 in Canada affirmed that, were it not for their family and friends, they would have experienced difficulty in finding help [7]. Moreover, the stigma surrounding mental illness can lead to treatment avoidance, delays to care, and discontinuation of treatment, leading to a lack of proper expert diagnosis and support [8].

For these reasons, there is burgeoning interest in utilizing automated means to help bridge the gap between at-risk persons and mental health services. In particular, in Computational Linguistics, the use of Internet fora and social media to discuss these matters offers an opportunity to devise methods of mental status assessment from text. As such, there are

${ }^{*}$ meurs.marie-jean@uqam.ca

This article is (C) 2021 by author(s) as listed above. The article is licensed under a Creative Commons Attribution (CC BY 4.0) International license (https://creativecommons.org/licenses/by/4.0/legalcode), except where otherwise indicated with respect to particular material included in the article. The article should be attributed to the author(s) identified above. 
increasing research efforts towards risk detection of mental health problems and self-harming behaviors [9-11].

However, for these analyses to be fully useful to the diagnostic and treatment processes, the predictions made must be as informative as possible. This can be broadly construed as a matter of interpretability. In doing so, recent work has been aimed in this direction [12, 13]. Nonetheless, we contend that the primary concern should be actionability. That is, the predictions should contain information that is clinically meaningful, i.e., pertaining to symptoms.

Thus, the work presented focuses on the dataset put forth by Losada, Crestani, and Parapar [14]. This corpus is composed of textual content written on Reddit by 90 social media users, paired with a standard questionnaire covering signs and symptoms of depression, the Beck Depression Inventory-Second Edition (BDI-II) [15]. While detailed, this dataset is of modest size, making it difficult to learn the mapping from text to BDI-II answers directly. Instead, the prediction of answers is based on those of known persons in a nearest-neighbor fashion.

The main contributions of this work are:

(1) Comparing the effectiveness in predicting BDI-II answers of two methods of establishing similarity. The first is based on the unsupervised extraction of topics from textual productions (TPs). The second consists of explicitly learning similarity as a matter of authorship attribution through the use of neural encoders.

(2) Measuring whether the dependencies between different aspects of depression (as per the BDI-II) can be leveraged in such a framework.

The paper is organized as follows. Section 2 presents the prediction algorithm as well as the similarity measures used, and similar work in the literature. Section 3 describes the evaluation metrics, the experimental settings as well as the results obtained. Finally, Section 4 discusses our findings and Section 5 concludes this paper.

\section{Resources and Methodology}

\subsection{Dataset and BDI-II structure}

As previously mentioned, the dataset proposed by Losada, Crestani, and Parapar [14] is composed of the TP 90 Reddit users, each one being paired with BDI-II answers. The number of writings per person ranges from 12 to 1164 (median of 234). These writings range in word count from 11 to 1515 (median of 34).

The BDI-II is a 21-item self-report measure of depressive symptoms experienced during the past week (see questions and possible answers provided in the appendix). It covers different aspects of the manifestation of depression, related to affective, cognitive, somatic and vegetative symptoms, according to the DSM-IV [16] criteria for major depression ${ }^{1}$. Each item is associated with a single symptom allowing self-assessment of a specific behavioral manifestation of depression. Items are ranked to reflect the range of severity of the symptom from 0 (absent) to 3 (severe). The BDI-II covers symptoms range from the very concrete, such as changes in sleep pattern and appetite, to more abstract aspects, such as guilt or punishment feelings. Depression severity is scored from the BDI-II by adding the ratings for all the 21 items, obtained from self-assessment. The minimum score is 0 and maximum score is 63 , with higher scores indicating greater symptom severity. Depression levels are divided in 4 categories: minimal (scores of 0-13), mild (14-19), moderate (20-28) and severe (29-63).

\footnotetext{
${ }^{1}$ The American Psychiatry Association [17] reports that the core criterion symptoms applied to the diagnosis of a major depressive episode has not changed from DSM-IV to DSM-V, making the BDI-II valid for both versions.
} 


\begin{tabular}{|c|c|c|c|c|c|}
\hline & $v_{1}$ & $v_{2}$ & $v_{3}$ & $v_{4}$ & \\
\hline$\alpha\left(v_{i}, u\right)$ & 0.4 & 0.2 & 0.1 & 0.3 & $u$ \\
\hline Q1 & 0 & 0 & 3 & 1 & $\rightarrow 0$ \\
\hline Q2 & 0 & 1 & 2 & 1 & $\rightarrow 1$ \\
\hline Q3 & 1 & 0 & 2 & 3 & $\rightarrow 1$ \\
\hline : & & & & & \\
\hline . & & & & & \\
\hline
\end{tabular}

Figure 1. Prediction example on BDI-II answers from person $u$ based on the similarity $\alpha\left(v_{i}, u\right)$ between the TPs of persons $v_{i}: i=1, \ldots, 4$ and $u$. Lines 2 to 4 indicate values of the BDI-II answers given by persons $v_{i}$ to questions Q1 to Q3. At each question, the answer with highest total score is selected.

The BDI-II was filled out by the 90 participants immediately before the collection of their TPs. Two key difficulties of this dataset are the limited amount of annotated observations as well as their large size in terms of textual content. However, to our knowledge, this dataset is the first containing a clinically meaningful, detailed inventory of signs of depression.

\subsection{Similar textual production, similar potential symptoms?}

With a sound measure of similarity between the TP of different persons, one could predict a new person's answers to the BDI-II based on those of a pool of known persons, using the $\mathrm{TP}$ similarity to weight their respective importance. Let $\alpha(u, v) \in[0,1]$ be such a similarity between the TPs of persons $u$ and $v$, with a greater number denoting greater similarity. Let $A$ be the set of answers to some question in the BDI-II, with $\mathbf{1}_{a}(v)$ indicating whether person $v$ selected answer $a \in A$. The predicted answer among $A$ for person $u$ given a pool of persons $V$ is given by:

$$
\underset{a \in A}{\operatorname{argmax}} \sum_{v \in V} \alpha(u, v) \mathbf{1}_{a}(v)
$$

In this way, there is no need to learn the map from persons' TPs to BDI-II answers but one can simply exploit the hypothesis that persons producing similar contents (as per the similarity measure) could present similar potential symptoms. This approach still allows for these persons to diverge in some answers, provided that others, less similar in their production, collectively agree, as illustrated in Fig 1. Furthermore, the answer prediction can be made based solely on a fixed number of nearest neighbors, or on the entire pool of persons.

However, no such similarity measure is available off-the-shelf. This work hence attempts to induce it considering two methods. The first one consists in extracting topics from TPs, then compare persons' TPs in the topic space. The second method approaches the task of learning similarity between persons' TPs as an authorship attribution task. That is, train a model to decide whether two sets of TPs were written by the same person or not.

\subsection{Topic extraction}

As the first method is based on unsupervised topic extraction, a reliable and robust approach is required to capture the underlying topic structure from depression related TPs. Latent Dirichlet Allocation (LDA) [18] was selected since it has been shown to be meaningful to depression symptomatology $[10,19,20]$. LDA allows the inference of topics (distributions over tokens) in an unsupervised manner. Once the topics are extracted from the concatenation of each person's TPs, the TPs can be represented as vectors of topics, which are then used to compute their similarity. Similarly, TF-IDF is used as a baseline [21]. 


\subsection{Authorship attribution}

As mentioned, the TPs of persons in the dataset can be large both in number of documents and in length of documents. Moreover, they vary in both of these respects. For these reasons, we require an encoding architecture that is computationally efficient and invariant to the quantity of text encoded. Therefore, Deep Averaging Networks (DANs) [22] are considered for encoding TPs. DANs build a dense encoding of a document by averaging together the vector representations of the tokens making up the document and feeding it through a deep feed-forward network. Here, tokens are represented as one-hot vectors. The average is then equivalent to a token-frequency representation of documents. This allows for efficient preprocessing of documents and a normalized representation, which would be less sensitive to document or word count. The DAN thus can transform the entirety of a person's TP into a vector representation used to compute the similarity.

The encoder itself is trained in an unsupervised manner. Pairs of persons are drawn at random with replacement, i.e. the pair may contain the same person twice or different persons. The encoder must decide whether the associated TPs were written by the same person or not. To avoid overfitting, regularization in the form of dropout is applied, both in documents and in length of documents. Specifically, a small set of each person's TP is sampled to feed to the encoder. Crucially, a new sample is taken at every training step. Similarly, word dropout [22] is applied on the tokens in the Bag-of-Words (BoW) representation. In validation, however, the entire TP is considered.

\subsection{Alternative approaches}

There has been substantial work in predicting the risk for mental health issues or selfharming behaviors from text. Nonetheless, these predictions are usually of degree of risk, either binary [23], or with several levels [24]. Some recent efforts have focused on attempting to signal the portions of text that most contribute to the prediction, thus potentially offering some details on the manifestation of the issue.

The current state of the art for a variety of natural language processing challenges [see 25] has been dominated by Transformer-based approaches [26]. Nonetheless, such approaches are ill-fitted here. They can be computationally intensive and offer no clear manner of aggregating groups of documents. In contrast, DANs can be applied directly to token-count representations, greatly reducing processing time.

As for prediction of BDI-II answers, the eRisk 2019 and 2020 shared tasks resulted in a growing interest from the research community [27-31]. Some of these involve direct learning of textual psycho-linguistic features relevant to depression [28, 30]. Other direct approaches involve the use of pre-trained Transformers [31]. To address the computational difficulty of handling long TPs, the authors propose truncating or splitting documents into more manageable lengths. Nonetheless, this presupposes that any portion of a person's TP is pertinent to their depression symptoms or lack thereof. An approach comparable to this work, taken by [32] sought to compare the textual output of persons and the text contained in the BDI-II questionnaire. However, the reported results are modest, mainly due to the brevity of the questionnaire text.

\section{Experiments}

\subsection{Metrics}

Losada, Crestani, and Parapar [14] propose four metrics to evaluate the prediction of BDIII answers. As mentioned, the questionnaire is composed of 21 questions with 4 possible answers (from 0 to 3), except for questions 16 and 18, where there are seven possible 
answers $(0,1 \mathrm{a}, 1 \mathrm{~b}, 2 \mathrm{a}, 2 \mathrm{~b}, 3 \mathrm{a}, 3 \mathrm{~b})$. The metrics, described hereafter, aim to evaluate exact correspondence, per-item closeness and overall closeness.

- The Average Hit Rate (AHR) measures the proportion of exactly correct predictions. If the hit rate for a given person is defined as the proportion of questions for which the prediction matched exactly the true answer, the AHR is the mean hit rate across all persons.

- The Average Closeness Rate (ACR), by contrast, measures how close the predicted answers were to the true answer. The absolute differences between the value of the predicted and true answers are computed. For questions 16 and 18, each pair (1a,1b), $(2 \mathrm{a}, 2 \mathrm{~b}),(3 \mathrm{a}, 3 \mathrm{~b})$ is considered to have the same value. Then, the absolute difference, $a d$, is compared to the maximum possible absolute difference, 3 , to give the closeness rate, $C R=\frac{3-a d}{3}$. The closeness rate for a person is the mean closeness rate across questions. The ACR is then simply the mean closeness rate across persons.

- The Average Difference between Overall Depression Levels (ADODL) measures the difference in total score. As previously mentioned, the answers to each question in the BDI-II are summed up to compute an overall depression level. The Difference between Overall Depression Levels (DODL) is simply the absolute difference between this total as per the ground truth and as per the predicted answers, normalized, $D O D L=\frac{63-a d}{63}, 63$ being the maximum possible depression level. As with the previous metrics, the ADODL is the mean DODL over all persons. As previously mentioned, depression levels as measured by the BDI-II can be assigned to one of four categories.

- The Depression Category Hit Rate (DCHR), is the accuracy of the categorization afforded by the predicted BDI-II.

\subsection{Similarity learning}

All models are trained on data collected by Losada, Crestani, and Parapar [23]. The dataset consists of TPs on Reddit fora from 1707 persons, who are categorized as either at risk of depression or not. For each approach, two forms of tokenization are used in separate models: in-word character trigrams and word stemming. Tokens used by fewer than 25 persons are excluded. The labels are ignored.

The inverse document frequencies and LDA topics are computed using each person's TP as a single document. The LDA model was built with Gensim, trained over 50 iterations with the Variational Bayes method. The number of topics was empirically derived. The best results are often acquired when performing LDA around 50 topics in a depression-related context [19]. For this reason, several models with varying numbers of topics were trained, to discover that using LDA with 30 topics on this specific dataset gave the leading performance. Training both models (trigrams and stems) took about 90 minutes on a $2.8 \mathrm{GHz}$ Intel Core i5 CPU with 8GB of memory.

As for DANs, the training considerations are as follows. Firstly, note that the number of positive unordered pairs of TPs (same person) grows linearly with the number of persons, while the number of negative ones (different persons) grows quadratically. There is no prior reason to favor positive or negative pairs in training the encoder. Therefore, the negative class is undersampled in training, generating positive and negative pairs in equal amounts. In validation, however, it is relevant to reduce the variance brought on by validating on any particular set of negative pairs. Therefore, all possible unordered pairs are generated, validating on the same set of pairs at each epoch. Thus, the balanced accuracy is monitored on a $10 \%$ validation set in order to perform early stopping [33]. This is computed as the unweighted average recall of each of the two classes.

Secondly, to avoid overfitting, in training, only a sample of 20 documents is taken from each person's TP. Persons having produced fewer than 50 documents are excluded. When 
Table 1. Results of the proposed models on BDI-II prediction with persons split into training and test as per Losada, Crestani, and Parapar [14]. The BDI-II predictions were tested considering the $k=\{3,5,7,9,-\}$ nearest neighbors ('-' for all). The results are the best for each metric attained by the model, with the corresponding $k$ in parentheses. The bottom of the Table presents the best results obtained at eRisk 2020 for each metrics.

\begin{tabular}{|l|c|cccc|}
\cline { 3 - 6 } \multicolumn{2}{c|}{} & \multicolumn{4}{c|}{ BDI-II } \\
\hline Model & Tokenization & AHR & ACR & ADODL & DCHR \\
\hline TF-IDF & trigrams & $\mathbf{. 3 9 6}(9)$ & $\mathbf{. 7 0 1}(3)$ & $.812(-)$ & $.333(-)$ \\
TF-IDF & stems & $.391(9)$ & $.697(3)$ & $.810(-)$ & $.348(9)$ \\
LDA & trigrams & $.364(-)$ & $.689(-)$ & $.815(-)$ & $.261(5)$ \\
LDA & stems & $.380(-)$ & $.696(-)$ & $\mathbf{. 8 3 2}(-)$ & $\mathbf{. 4 2 0}(9)$ \\
DAN (small) & trigrams & $.386(-)$ & $.698(-)$ & $.823(5)$ & $.333(9)$ \\
DAN (small) & stems & $.364(-)$ & $.689(-)$ & $.813(-)$ & $.348(5)$ \\
DAN (large) & trigrams & $.380(-)$ & $.694(-)$ & $.820(5)$ & $.333(3)$ \\
DAN (large) & stems & $.374(-)$ & $.695(-)$ & $.824(-)$ & $.319(3)$ \\
\hline \hline Zeroes & & .364 & .644 & .644 & .145 \\
Ones & & .310 & .735 & .819 & .246 \\
Random & & .232 & .586 & .753 & .271 \\
\hline \hline BioInfo@UAVR [28] & & $\mathbf{. 3 8 3}$ & .692 & .760 & .300 \\
iLab [31] & & .371 & $\mathbf{. 6 9 4}$ & .817 & .271 \\
prhlt [30] & & .346 & .674 & .806 &. $\mathbf{3 5 7}$ \\
relai [29] & & .364 & .683 & $\mathbf{. 8 3 2}$ & .343 \\
\hline
\end{tabular}

matching a person's TP against itself (positive pair), the samples are mutually exclusive. Moreover, word dropout (.15) is applied on the tokens, independently so across TP samples. The encoders are trained by gradient descent, using the Adam algorithm [34] over 50 epochs and performing early stopping as per the balanced accuracy on the validation set. The models were built with Tensorflow. One large and one small model were trained for each tokenization scheme. Large models consist of 9 layers of 1024 units whereas small ones consist of 9 layers of 512 units. This results in large models with $25.1 \mathrm{M}$ and $23.1 \mathrm{M}$ parameters for stems and trigrams, respectively, and small models of $10.4 \mathrm{M}$ and $9.4 \mathrm{M}$ parameters, likewise respectively. All layers, including the final one, use rectified linear unit activation. All models were initialized by He normal initialization [35] and trained with mini-batches of size 32. The total running time for all four models was about 3 hours on a $3.6 \mathrm{GHz}$ Intel Core i7-7700 CPU with $31.2 \mathrm{~GB}$ of memory. The balanced accuracies achieved in validation were .836 for the small trigram model, .859 for the small stem model, .852 for the large trigram model and .834 for the large stem model.

\subsection{Prediction of BDI-II answers}

Once the encoders are trained, the BDI-II answers of persons is predicted based on similarity to a subset of the TP of persons whose BDI-II is known. The similarity itself is computed as the cosine similarity between the encoded TPs. These encodings being positive, the cosine similarity is in the unit interval. The dataset, as put forth by Losada, Crestani, and Parapar [14], is split into the TPs of 20 persons for training and the TPs of 70 persons for test. However, the distribution of BDI-II scores are quite different between these two subsets: the number of persons in each of the four depression categories are $(4,4,4,8)$ for the training set and $(10,23,18,19)$ for the test set. Therefore, we report results on the original split as well as those obtained by stratified 5 -fold cross validation. The experiments are conducted basing prediction on the nearest 3, 5, 7 and 9 neighbors as well as the entire pool for prediction. These results are compared to those obtained by answering only 0 or only 1 (the most frequent answers) to each question, as well as uniform random prediction averaged over 100 iterations. 
Table 2. Comparing the results of the proposed models on the BDI-II prediction in 5fold cross validation and authorship decision tasks. The BDI-II predictions were tested considering the $k=\{3,5,7,9,-\}$ nearest neighbors ('-' for all). The results are the best for each metric attained by the model, with the corresponding $k$ in parentheses. For authorship, in addition to the accuracy, we report the average similarity score produced by the model for negative and positive pairs of documents. For reference, the results of predicting only 0 , only 1 or a uniformly random answer to each question are provided.

\begin{tabular}{|l|c|cccc|ccc|}
\cline { 3 - 9 } \multicolumn{2}{c|}{} & \multicolumn{9}{c|}{ BDI-II } & \multicolumn{3}{c|}{ Authorship } \\
\hline Model & Tokenization & AHR & ACR & ADODL & DCHR & pos sim. & neg sim. & acc \\
\hline TF-IDF & trigrams & $.378(-)$ & $.697(7)$ & $.805(3)$ & $.311(3)$ & $.906 \pm .132$ & $.852 \pm .126$ & .509 \\
TF-IDF & stems & $.381(-)$ & $.670(9)$ & $.807(3)$ & $.278(9)$ & $.825 \pm .193$ & $.821 \pm .164$ & .495 \\
LDA & trigrams & $.387(-)$ & $.698(-)$ & $.794(9)$ & $.289(3)$ & $.763 \pm .285$ & $.361 \pm .280$ & .760 \\
LDA & stems & $.386(-)$ & $.670(-)$ & $.799(-)$ & $.322(3)$ & $.863 \pm .204$ & $.446 \pm .291$ & .761 \\
DAN (small) & trigrams &. $\mathbf{4 0 6}(-)$ & $\mathbf{. 7 1 3 ( - )}$ & $\mathbf{. 8 2 4}(7)$ & $\mathbf{. 3 8 8}(7)$ & $.878 \pm .188$ & $.445 \pm .301$ & .746 \\
DAN (small) & stems & $.396(-)$ & $.709(-)$ & $.816(5)$ & $.378(5)$ & $.849 \pm .211$ & $.346 \pm .284$ &. $\mathbf{8 0 4}$ \\
DAN (large) & trigrams & $.396(-)$ & $.710(-)$ & $.797(7)$ & $.356(3)$ & $.911 \pm .148$ & $.567 \pm .323$ & .678 \\
DAN (large) & stems & $.403(-)$ & $\mathbf{. 7 1 4}(-)$ & $.804(9)$ & $.310(9)$ & $.852 \pm .205$ & $.378 \pm .297$ & .788 \\
\hline \hline Zeroes & & .359 & .636 & .636 & .156 & & & \\
Ones & & .302 & .730 & .814 & .244 & & & \\
Random & & .229 & .584 & .758 & .284 & & & \\
\hline
\end{tabular}

Table 1 presents the results in prediction of BDI-II answers using the original trainingtest split, including the best results obtained at eRisk 2020 for the sake of comparison. Table 2 presents the results on cross-validated BDI-II prediction. Furthermore, Table 2 shows the results of our different models on authorship decision, notwithstanding that the TF-IDF and LDA models were not trained for this task. These results are computed on the entire dataset (TPs of 90 persons). Negative pairs are generated using the entire TP of each person. Positive pairs are generated by partitioning each person's TP in half. These partitions are resampled enough times to arrive at roughly equal numbers of positive and negative pairs.

Table 3. Mode of the answers to each question in the dataset (90 users).

\begin{tabular}{|l|c|c|c|c|c|c|c|}
\hline question & Q1 & Q2 & Q3 & Q4 & Q5 & Q6 & Q7 \\
\hline mode & 1 & 1 & 1 & 1 & 0 & 0 & 0 \\
\hline \hline question & Q8 & Q9 & Q10 & Q11 & Q12 & Q13 & Q14 \\
\hline mode & 0 & 0 & 0 & 0 & 1 & 0 & 0 \\
\hline \hline question & Q15 & Q16 & Q17 & Q18 & Q19 & Q20 & Q21 \\
\hline mode & 1 & $1 b$ & 0 & 0 & 0 & 1 & 0 \\
\hline
\end{tabular}

\section{Discussion}

The proposed models achieve interesting results both in cross validation (Table 2) and on the original split (Table 1), indicating that the authorship decision task can induce a similarity measure useful for depression symptom detection. As shown in Table 1, these models achieve results comparable to those reported by Losada, Crestani, and Parapar [14] on the original split, with the best model surpassing the previous best DCHR. While authorship seems a good proxy for predicting BDI-II answers, the converse does not hold, as the TF-IDF model performs quite poorly in authorship attribution, as shown in Table 2. Upon closer examination, on the cross-validation experiments, TF-IDF models predict always the same BDI-II answers when considering a large number of neighbors. This is not the case for the other models.

The BDI-II answers predicted by TF-IDF are very close to the mode of the answers to each question in the dataset of 90 users, reported in Table 3. As a consequence, TF-IDF 
Table 4. Results of the proposed models on predicting BDI-II answers in five fold cross validation using Dependent Multilabel k-Nearest Neighbors algorithm (DMkNN) and Multilabel k-Nearest Neighbors algorithm (MLkNN). The predictions of BDI-II answers were tested considering the $(k, \delta) \in\{(5,3),(7,5),(9,5),(20,10),(30,10),(40,10)\}$ for DMkNN and $k \in\{5,7,9,20,30,40\}$ for MLkNN. The smoothing parameter for DMkNN, $s$, is set to 1 . The results are the best for each metric attained by the model, with the corresponding value(s) of $k$ in parentheses.

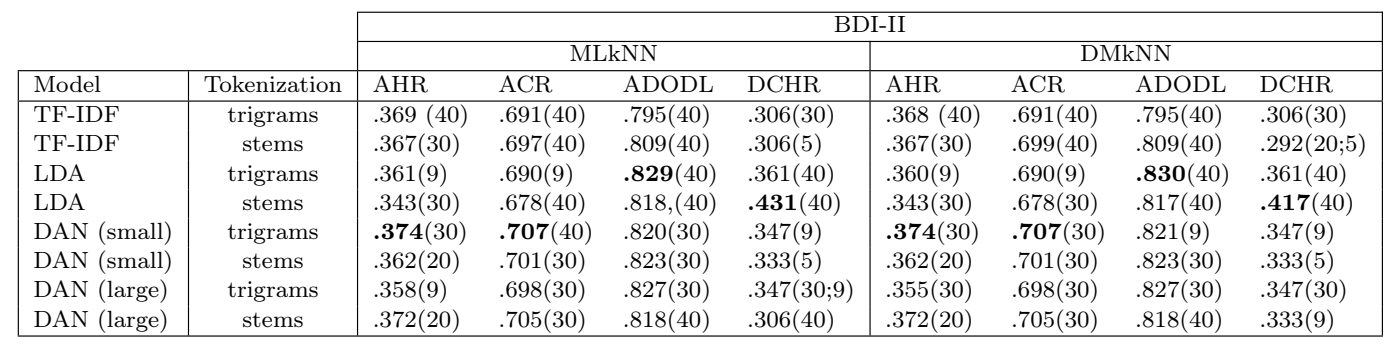

performs well on average, especially on distance-based metrics, such as ACR and ADODL. This is reflected by the proximity in ACR and ADODL of predicting 1 to all questions, which is also close to the mode of the answers to each question. On the original split, where the pool of known persons is limited, the BDI-II answers predicted by the TF-IDF vary. This sensitivity to the pool of known persons is reflected by the similarity computed in the authorship attribution task. As shown in Table 2, TF-IDF models produce the smallest range of similarity scores when shown excerpts from different persons. Obviously, the authorship attribution task is a proxy. By the very premise of the algorithm predicting BDI-II answers, the TPs compared never issue from the same person. What is interesting is the expressiveness of the similarity function, which requires, at a minimum, a wide range of values. This explains why, with all persons in the pool within a small range of similarity, numbers win out, and the answers predicted are the most common ones. It also points to a clear, inherent limitation of the approach at large. Collapsing the similarity into a single scalar expresses only if two persons produce similar content and not how. The aspects of similarity are instead expected to appear by comparing to the BDI-II answers of different persons.

The more basic issue with this premise is that, while the computed similarity between persons' TPs remains the same, their similarities as per the BDI-II answers vary in many ways. Firstly, the answers are differently spread out for each question. The standard deviation for each question over all filled BDI-II ranges from 0.8 for question 1 to 1.16 for question 7. Secondly, the variation changes with overall score. This is illustrated in Figure 2. When grouping persons by depression category, the spread on each question varies between categories. For example, answers from persons in the minimal depression category present a null standard deviation for questions 6,13 and 14. On the other hand, answers to questions 10 and 21 present a standard deviation among the moderately and severely depressed close to that of a uniform distribution $(\sqrt{5} / 2)$. Moreover, there are dependencies present between questions in the BDI-II, as evidenced by the heatmap in Figure 3.

For these reasons, further experiments were conducted with the Dependent Multilabel kNearest Neighbors algorithm (DMkNN) [36] algorithm, using the same similarities between TPs already computed. This algorithm verifies the agreement in other variables among the neighbors of the test instances as well as those of the pool of known instances. To alleviate the difficulty in finding complete agreement, the algorithm uses a fuzziness hyperparameter, $\delta$. Given that each of four or seven possible answers is treated as a separate class, larger values of $\delta$ are used than a binary setting would require. As $\delta<k$, these experiments required a larger pool of observations, and thus were not carried out on the 


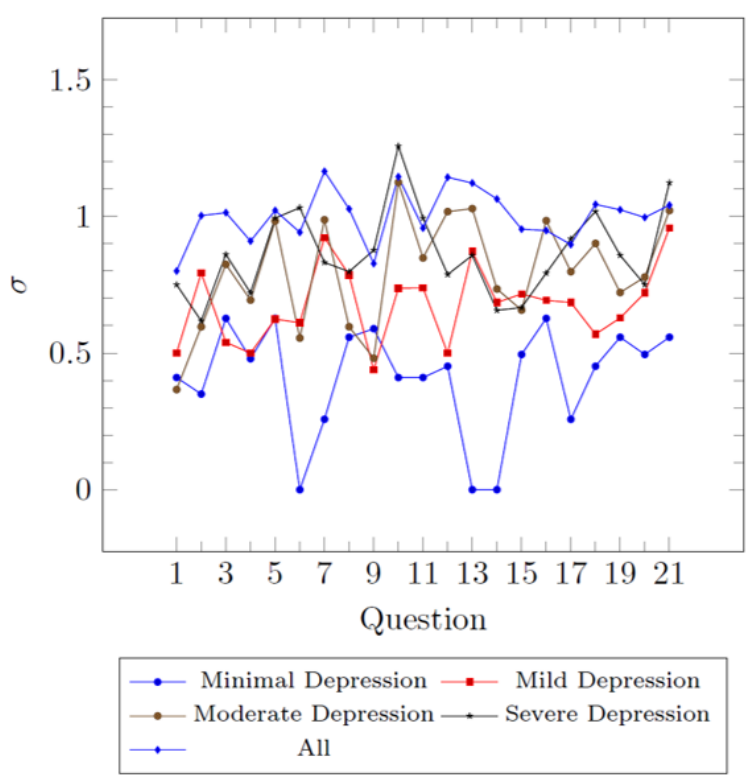

Figure 2. Standard deviation in answers to the BDI-II among persons in each depression category

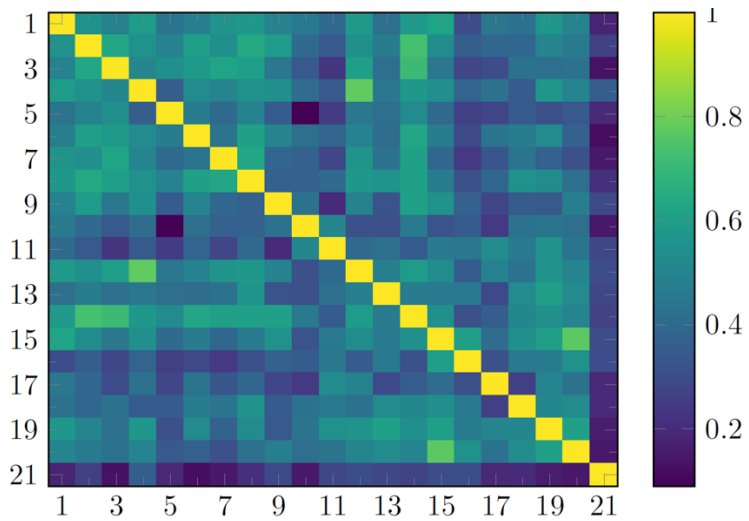

Figure 3. Pearson correlation coefficient between questions on the BDI-II among the 90 persons in the dataset

original training-test split put forth by Losada, Crestani, and Parapar [14]. This algorithm was also compared to Multilabel k-Nearest Neighbors algorithm (MLkNN) [37], which does not consider dependencies between output variables. As shown in Table 4, the results are comparable to those obtained by the first approach, with a slight increase in ADODL and a small decrease in AHR. As in the previous experiments, the models seem to favor larger values of $k$. This is consistent with the high variation in answers to several questions discussed earlier. There seems to be little difference in performance between the MLkNN and DMkNN algorithms both overall and in each model. One possible shortcoming of DMkNN in this context is the requirement of exact correspondence in the answers to other questions. However, relaxing this into a proximity requirement might encounter the same smoothing issues arising from aggregating too many neighbors.

An additional limitation of the approach thus far is that the similarity between TPs is computed on their entirety. By aggregating over the entire TPs, some localized similarities 
Table 5. Results of the proposed models on predicting BDI-II answers in five fold cross validation using $\mathrm{DMkNN}$ and MLkNN.The results are the best for each metric attained by the model, with the corresponding value of $(k, \delta, D, t)$ in parentheses.

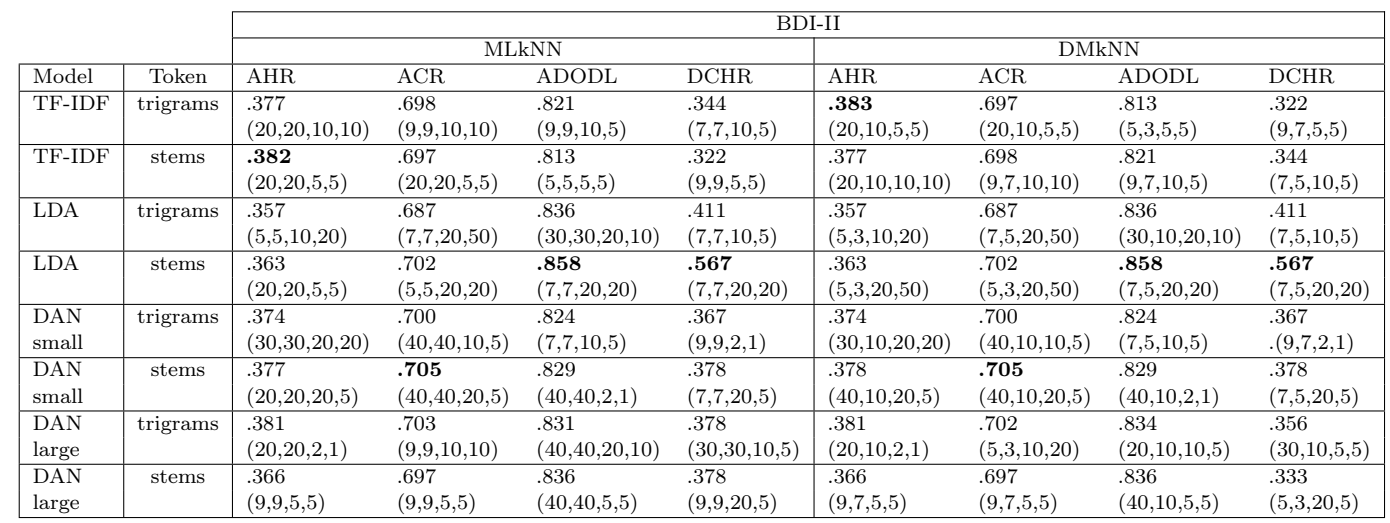

might be lost, where a finer-grained approach could find them. Arranging the writings in each TP in chronological order, we partition them into $D$ parts such that all the partitions in a TP are of roughly equal word count. We compute a finer-grained similarity by pairing each of the partitions of the TP considered and averaging over the highest $t$ similarities. Table 5 reports the results obtained in cross validation by the DMkNN and MLkNN algorithms using this similarity, with $(D, t) \in\{(2,1),(5,5),(5,10),(10,5),(10,10),(10,20),(20,10),(20,20)$, $(20,50)\}$. Performance improves overall across all metrics. All encoders appear to favor larger values of $D$ in most metrics. Nonetheless, larger values of $D$ incur greater computational complexity, both in the form of separate calls to the relevant encoders and in terms of comparisons, which grow quadratically with respect to $D$.

\section{Conclusion}

This work studied an approach to predict the potential answers to the BDI-II of a person from similarity measures between the textual productions of different persons. In particular, the experiments sought to compare the effectiveness of two methods of establishing similarity: using unsupervised extraction of topics and authorship attribution through the use of neural encoders. The proposed system achieves interesting results, indicating that the authorship attribution task can induce a similarity measure useful for depression symptom detection. The task proved difficult nonetheless, as a single similarity measure must account for the prediction of different signs and symptoms of depression. For these reasons, future work will be aimed at adapting the similarity between textual productions to the aspect of depression considered.

Reproducibility. The source code of the proposed systems is licensed under the GNU GPLv3. The datasets are provided on demand by the eRisk organizers.

\section{Acknowledgements}

This research was enabled in part by support provided by Calcul Québec and Compute Canada. MJM acknowledges the support of the Natural Sciences and Engineering Research Council of Canada [NSERC Grant number 06487-2017] and the Government of Canada's New Frontiers in Research Fund (NFRF), [NFRFE-2018-00484]. 


\section{References}

[1] S. L. James, D. Abate, K. H. Abate, S. M. Abay, C. Abbafati, N. Abbasi, H. Abbastabar, F. Abd-Allah, J. Abdela, A. Abdelalim, et al. "Global, Regional, and National Incidence, Prevalence, and Years Lived with Disability for 354 Diseases and Injuries for 195 Countries and Territories, 1990-2017: A Systematic Analysis for the Global Burden of Disease Study 2017". In: The Lancet 392.10159 (2018), pp. 1789-1858.

[2] World Health Organization et al. Mental Health Action Plan 2013-2020. Tech. rep. World Health Organization, 2013.

[3] D. E. Bloom, E. Cafiero, E. Jané-Llopis, S. Abrahams-Gessel, L. R. Bloom, S. Fathima, A. B. Feigl, T. Gaziano, A. Hamandi, M. Mowafi, et al. The Global Economic Burden of Noncommunicable Diseases. Tech. rep. Program on the Global Demography of Aging, 2012.

[4] Substance Abuse and Mental Health Services Administration. Key Substance Use and Mental Health Indicators in the United States: Results from the 2017 National Survey on Drug Use and Health. 2018.

[5] Canadian Mental Health Association. Fast Facts about Mental Illness. 2020.

[6] X. Qin, S. Wang, and C.-R. Hsieh. "The Prevalence of Depression and Depressive Symptoms among Adults in China: Estimation Based on a National Household Survey". In: China Economic Review 51 (2018), pp. 271-282.

[7] Statistics Canada. Care Counts: Receiving Care for a Mental Illness, 2018. 2020.

[8] C. Henderson, S. Evans-Lacko, and G. Thornicroft. "Mental Illness Stigma, Help Seeking, and Public Health programs". In: American Journal of Public Health 103.5 (2013), pp. 777-780.

[9] H.-C. Shing, S. Nair, A. Zirikly, M. Friedenberg, H. Daumé III, and P. Resnik. "Expert, Crowdsourced, and Machine Assessment of Suicide Risk via Online Postings". In: Proceedings of the Fifth Workshop on Computational Linguistics and Clinical Psychology: From Keyboard to Clinic. New Orleans, LA: Association for Computational Linguistics, 2018.

[10] G. Coppersmith, M. Dredze, C. Harman, K. Hollingshead, and M. Mitchell. "CLPsych 2015 Shared Task: Depression and PTSD on Twitter". In: Proceedings of the 2nd Workshop on Computational Linguistics and Clinical Psychology (CLPsych): From Linguistic Signal to Clinical Reality. 2015, pp. 31-39.

[11] D. Maupomé, M. Queudot, and M.-J. Meurs. In:

[12] J. Ive, G. Gkotsis, R. Dutta, R. Stewart, and S. Velupillai. "Hierarchical Neural Model with Attention Mechanisms for the Classification of Social Media Text Related to Mental Health". In: Proceedings of the Fifth Workshop on Computational Linguistics and Clinical Psychology: From Keyboard to Clinic. New Orleans, LA: Association for Computational Linguistics, 2018.

[13] H.-C. Shing, P. Resnik, and D. W. Oard. "A Prioritization Model for Suicidality Risk Assessment". In: Proceedings of the 58th Annual Meeting of the Association for Computational Linguistics. 2020, pp. 8124-8137.

[14] D. E. Losada, F. Crestani, and J. Parapar. "Overview of eRisk 2020: Early Risk Prediction on the Internet". In: Experimental IR Meets Multilinguality, Multimodality, and Interaction Proceedings of the Eleventh International Conference of the CLEF Association (CLEF 2020). Ed. by A. Arampatzis, E. Kanoulas, T. Tsikrika, S. Vrochidis, H. Joho, C. Lioma, C. Eickhoff, A. Névéol, L. Cappellato, N. Ferro (eds). Springer International Publishing, 2020.

[15] A. T. Beck, R. A. Steer, G. K. Brown, et al. "Beck Depression Inventory - Second edition manual". In: San Antonio, TX: Psychological Corporation 1 (1996), p. 82.

[16] C. C. Bell. "DSM-IV: diagnostic and statistical manual of mental disorders". In: Jama 272.10 (1994), pp. 828-829.

[17] American Psychiatry Association. Highlights of Changes from DSM-IV-TR to DSM-5. 2013.

[18] D. M. Blei, A. Y. Ng, and M. I. Jordan. "Latent Dirichlet Allocation". In: Journal of machine Learning research 3.Jan (2003), pp. 993-1022.

[19] P. Resnik, W. Armstrong, L. Claudino, T. Nguyen, V.-A. Nguyen, and J. Boyd-Graber. "Beyond LDA: Exploring Supervised Topic Modeling for Depression-related Language in Twitter". In: Proceedings of the 2nd Workshop on Computational Linguistics and Clinical Psychology: From Linguistic Signal to Clinical Reality. 2015, pp. 99-107.

[20] D. Maupomé and M.-J. Meurs. "Using Topic Extraction on Social Media Content for the Early Detection of Depression." In: CLEF (Working Notes). Vol. 2125. 2018. 
[21] H. C. Wu, R. W. P. Luk, K. F. Wong, and K. L. Kwok. "Interpreting TF-IDF Term Weights as Making Relevance Decisions". In: ACM Transactions on Information Systems (TOIS) 26.3 (2008), pp. 1-37.

[22] M. Iyyer, V. Manjunatha, J. Boyd-Graber, and H. Daumé III. "Deep Unordered Composition Rivals Syntactic Methods for Text Classification". In: Proceedings of the 53rd Annual Meeting of the Association for Computational Linguistics and the 7th International Joint Conference on Natural Language Processing. 2015, pp. 1681-1691.

[23] D. E. Losada, F. Crestani, and J. Parapar. "Overview of eRisk: Early Risk Prediction on the Internet". In: International Conference of the Cross-Language Evaluation Forum for European Languages. Springer. 2018, pp. 343-361.

[24] A. Zirikly, P. Resnik, O. Uzuner, and K. Hollingshead. "CLPsych 2019 Shared Task: Predicting the Degree of Suicide Risk in Reddit Posts". In: Proceedings of the Sixth Workshop on Computational Linguistics and Clinical Psychology. 2019, pp. 24-33.

[25] A. Wang, Y. Pruksachatkun, N. Nangia, A. Singh, J. Michael, F. Hill, O. Levy, and S. Bowman. "SuperGLUE: A Stickier Benchmark for General-Purpose Language Understanding Systems". In: Advances in Neural Information Processing Systems. 2019, pp. 3266-3280.

[26] A. Vaswani, N. Shazeer, N. Parmar, J. Uszkoreit, L. Jones, A. N. Gomez, Ł. Kaiser, and I. Polosukhin. "Attention Is All You Need". In: Advances in neural information processing systems. 2017, pp. 5998-6008.

[27] A. Trifan and J. L. Oliveira. "BioInfo@ UAVR at eRisk 2019: Delving into Social Media Texts for the Early Detection of Mental and Food Disorders." In: CLEF (Working Notes). 2019.

[28] L. Oliveira. "BioInfo@ UAVR at eRisk 2020: On the Use of Psycholinguistics Features and Machine Learning for the Classification and Quantification of Mental Diseases". In: CLEF (Working Notes). 2020.

[29] D. Maupomé, M. D. Armstrong, R. Belbahar, J. Alezot, R. Balassiano, M. Queudot, S. Mosser, and M.-J. Meurs. "Early Mental Health Risk Assessment through Writing Styles, Topics and Neural Models". In: CLEF (Working Notes). 2020.

[30] A.-S. Uban and P. Rosso. "Deep Learning Architectures and Strategies for Early Detection of Self-Harm and Depression Level Prediction". In: CLEF (Working Notes). 2020.

[31] R. Martınez-Castano, A. Htait, L. Azzopardi, and Y. Moshfeghi. "Early Risk Detection of Self-Harm and Depression Severity using BERT-based Transformers". In: CLEF (Working Notes). 2020.

[32] P. Abed-Esfahani, D. Howard, M. Maslej, S. Patel, V. Mann, S. Goegan, and L. French. "Transfer Learning for Depression: Early Detection and Severity Prediction from Social Media Postings". In: CLEF (Working Notes). 2019.

[33] J. D. Kelleher, B. Mac Namee, and A. D'arcy. Fundamentals of Machine Learning for Predictive Data Analytics: Algorithms, Worked Examples, and Case Studies. MIT press, 2020.

[34] D. P. Kingma and J. Ba. "Adam: A Method for Stochastic Optimization". In: arXiv preprint arXiv:1412.6980 (2014).

[35] K. He, X. Zhang, S. Ren, and J. Sun. "Delving Deep into Rectifiers: Surpassing HumanLevel Performance on ImageNet Classification". In: Proceedings of the IEEE International Conference on Computer Vision (ICCV).

[36] Z. Younes, F. Abdallah, T. Denoeux, and H. Snoussi. "A Dependent Multilabel Classification Method Derived from the k-Nearest Neighbor Rule". In: EURASIP Journal on Advances in Signal Processing 2011 (2011), pp. 1-14.

[37] M.-L. Zhang and Z.-H. Zhou. "ML-KNN: A Lazy Learning Approach to Multi-Label Learning". In: Pattern Recognition 40.7 (2007), pp. 2038 -2048. 